\title{
Implications of longitudinal muscle fibre splitting in neurogenic and myopathic disorders
}

\author{
M ICHAEL SW A S A N D MARTIN S. SCHWARTZ \\ From the Section of Neurological Sciences, The London Hospital, London
}

SU M MARY Histological and electromyographic studies indicate that longitudinal muscle fibre splitting is a common finding in neuromuscular disorders. Separated fragments derived by splitting may undergo degeneration or enlarge to become separate, innervated fibres, thus leading to an increased number of fibres within motor units. Splitting may, therefore, lead to the formation of clusters of fibres of uniform histochemical type, but of variable diameter and length, both in neurogenic and in myopathic disorders. Fibre splitting is thus a factor leading to functional compensation in these disorders.

Longitudinal splitting of muscle fibres has attracted little attention in the past (see Adams et al., 1962), probably because, in formalin-fixed tissue, it is difficult to distinguish true fibre splitting from artefactual cracking, caused by tissue shrinkage. Fibre splitting is a well-known feature of Duchenne and limb girdle muscular dystrophies (Dubowitz and Brooke, 1973), and of CharcotMarie-Tooth disease (Haase and Shy, 1960), but in other neuromuscular disorders it is said to be uncommon (Dubowitz and Brooke, 1973). However, in frozen material longitudinal fibre splitting can be recognised more easily, and it has recently become apparent that fibre splitting is present in other neuromuscular disorders (Cazzato, 1970; Schwartz et al., 1976; Swash et al., 1977). It is the purpose of this paper to review the histological and EMG evidence for fibre splitting in neuromuscular disease and to discuss the implications of the phenomenon for present concepts of the pathology and natural history of these disorders.

\section{Evidence for splitting}

HISTOLOGICAL EVIDENCE

Longitudinal fibre splitting does not occur in normal muscle, except near myotendinous insertions (Bell and Conen, 1968). In a single transverse section splitting can be recognised by the presence of a cleft, usually beginning from the

Address for reprint requests: Dr M. Swash, Section of Neurological Sciences, The London Hospital, London E1 1BB.

Accepted 18 July 1977

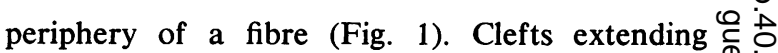
across the whole diameter of a fibre, dividing it $\stackrel{\leftrightarrow}{\sim} \vec{N}$ into two parts of identical histochemical type, can be recognised as splitting if the two parts are enclosed in a single endomysial tube or if, in consecutive transverse serial sections, the cleft extends as a fissure through only part of the fibre (Fig. 2). Fibre splitting is prominent in chronic neurogenic disorders, especially in Charcot-MarieTooth disease (Haase and Shy, 1973; Swash et al., 1977), in Kugelberg-Welander disease (Cazzato, 1970; Schwartz et al., 1976), and in some cases of motor neurone disease (Schwartz et al., 1976). Splitting also occurs in chronic polymyositis (Swash et al., 1977), and in Duchenne and limbgirdle dystrophies (Erb, 1891; Bell and Conen, 1968; Dubowitz and Brooke, 1973).

In muscle biopsies of 18 patients with neurogenic disorders fibre splitting was found in 15 . In three of these biopsy samples more than 20 split fibres, amounting to more than $5 \%$ of the fibres in the sample, were recognised in a single section (Swash et al., 1977). In neurogenic disorders splitting predominantly affects hypertrophied fibres, and in most instances (Figs. 2 and 3 ) individual clefts or splits are associated with a centrally placed sarcolemmal nucleus (Banker, 1960; Schwartz et al., 1976). Splitting may consist only of a single central or lateral fissure (Fig. 3), or it may lead to the formation of two or more apparently separate fibres of identical histochemical type (Fig. 4), each surrounded by a layer of basement membrane (Schwartz et al., 1976). Splitting affects type 1 fibres more commonly than 


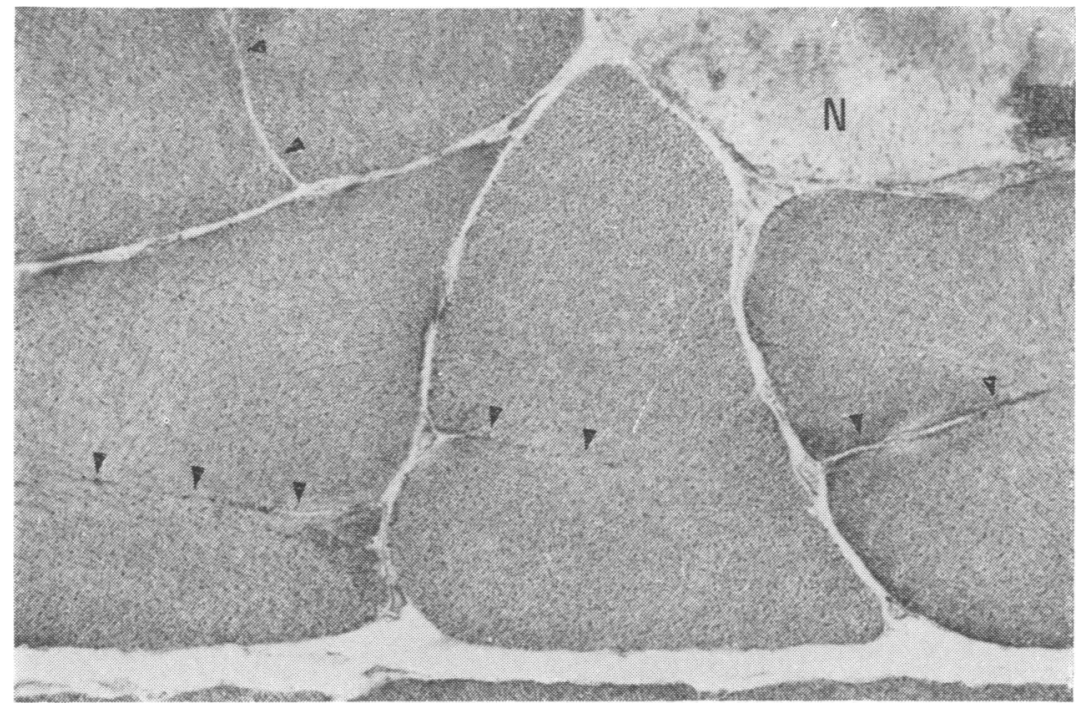

Fig. 1 Charcot-Marie-Tooth syndrome: quadriceps biopsy (transverse section) from a man aged 43 years. $N A D H \times 350$. Splitting (arrows) affects several adjacent hypertrophied type 1 fibres. There is a necrotic fibre nearby $(N)$.

type 2 fibres (Schwartz et al., 1976). In serial sections, these separate fibres may enlarge to attain a cross-sectional diameter similar to that of normal muscle fibres (Fig. 4). Others may fuse with each other, or insert onto adjacent interstitial connective tissue. It is likely that some split fibres become entirely separated from their parent fibre by this process, but in such instances it is difficult to be certain that the separate smaller fibre was derived by splitting from its neighbouring fibre. Bell and Conen (1968) aptly termed these fibres 'nesting fibres' (Fig. 3). Splitting also leads to the formation of very small separated fragments, some anucleate and non-viable, and others nucleate but probably too small to become functionally innervated (Schwartz et al., 1976).

In a study of the ultrastructural features (Fig. 5) of this process of splitting in neurogenic disorders, we have suggested that it results from stresses imposed on weakened muscles by normal weightbearing loads (Schwartz et al., 1976), a process similar to that shown experimentally in the rat (Hall-Craggs and Lawrence, 1970). James (1973), in a similar experiment in the rat, found that fibre fragments of this type were often faintly basophilic, and we have found histological evidence of active protein synthesis and of increase in size in such fragments of split fibres in chronic neurogenic disorders in man (Schwartz et al., 1976; Swash et al., 1977). The histological features of these split fibres, however, are not those of active subendomysial regeneration of the type discussed by Schmalbruch (1976) (see Figs. 1, 2, and 5).

We have found that, in the chronic form of polymyositis, hypertrophied fibres sometimes show splitting similar to that found in hypertrophied fibres in neurogenic disorders. In other fibres 'splitting' consists of extrusion of necrotic portions of damaged fibres, or is due to subendomysial regeneration from myoblasts formed after segmental necrosis, the sarcolemmal tube remaining intact (Schmalbruch, 1976; Swash et al., 1977). Splitting associated with fibre hypertrophy is not a feature of acute polymyositis. In this disorder 'splitting' is usually related to segmental fibre necrosis, or to regeneration.

Splitting of hypertrophied fibres in Duchenne dystrophy and in limb-girdle dystrophy is well recognised (Erb, 1891; Bell and Conen, 1968).

\section{ELECTROMYOGRAPHIC EVIDENCE}

In EMG and single fibre EMG investigations employing a trigger-delay line, late components, appearing more than about $10 \mathrm{~ms}$ after the initial action potential of a motor unit, have been found both in neurogenic disorders (Borenstein and Desmedt, 1973; Stålberg et al., 1975), and in muscular dystrophy (Desmedt and Borenstein, 1973, 1975; Stålberg et al., 1974). Since the oscilloscope is triggered by the first part of the complex action potential of a single motor unit, the late components in these studies probably 


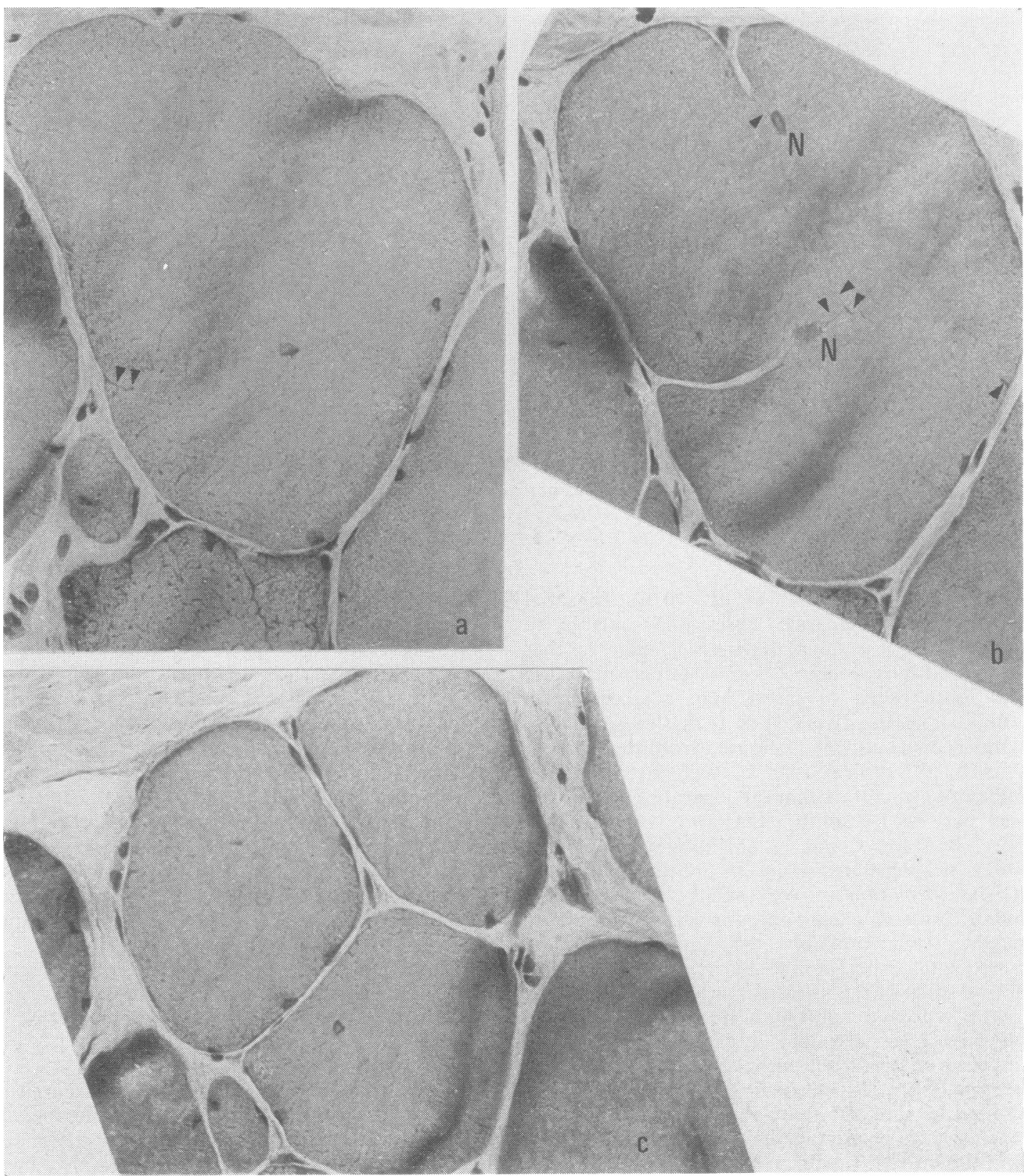

Fig. 2 Serial transverse sections of quadriceps. Kugelberg-Welander disease of at least 10 years duration in a 21 year old man. Haematoxylin and eosin $\times 560$. (a) A hypertrophied fibre, $250 \mu \mathrm{m}$ in diameter, contains a centrally-placed nucleus (arrows = splitting); (b) $22 \mu \mathrm{m}$ from (a). Two clefts, associated with central sarcolemmal nuclei $(N)$, invaginate the edge of the fibre (arrows=splitting); (c) $70 \mu \mathrm{m}$ from (a). The fibre has split into three apparently separate fibres. 


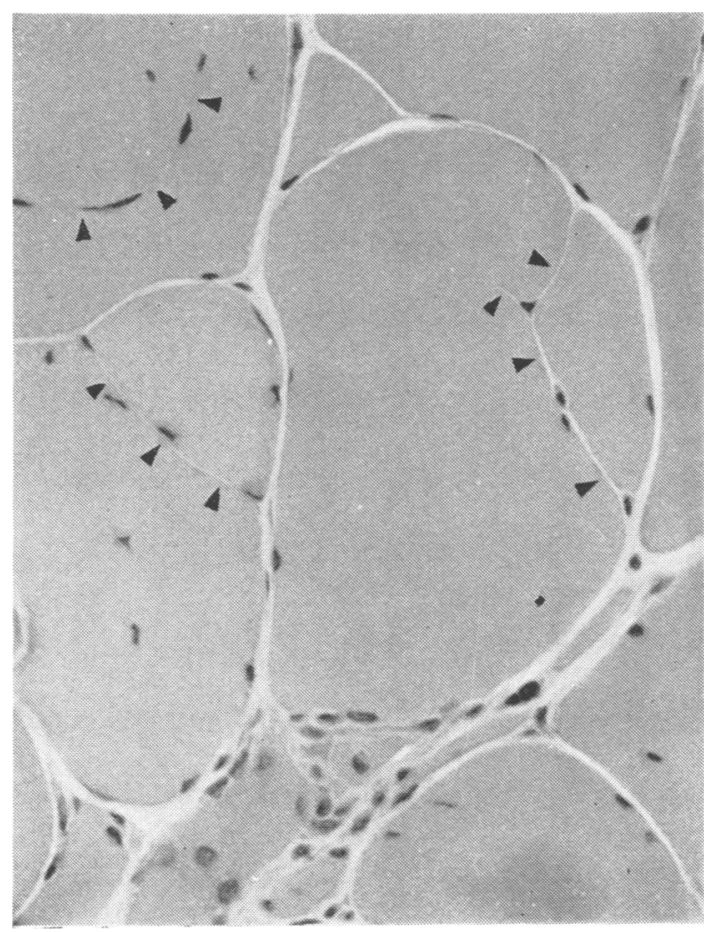

Fig. 3 Same biopsy as Fig. 1. Haematoxylin and eosin $\times 350$. Splitting (arrows), always associated with central sarcolemmal nuclei, is often complex in form. There is a small group of narrow, pointed (denervated?) fibres.

represent fibres innervated by collateral axon sprouts derived from the same motor unit. In neurogenic disorders these late components usually gave a greatly reduced propagation velocity (Stålberg et al., 1975), and similar late potentials with reduced propagation velocities have been found in Duchenne muscular dystrophy (Stålberg personal communication). The slowed propagation velocity of the late components of these abnormally complex motor unit potentials implies that these action potentials arise in fibres of small diameter. In neurogenic disorders the neuromuscular jitter of these late components is usually increased, with intermittent impulse blockings, suggesting that reinnervation of these small diameter fibres is functionally unstable (Stålberg $e t$ al., 1975). In some moderately advanced cases of Duchenne muscular dystrophy similar potentials with increased jitter and, rarely, with paired blocking, have been recorded (Stålberg et al., 1975; Schwartz et al., 1977), but the explanation of this phenomenon in this condition is, at present, uncertain.

\section{Implications in neuromuscular disease}

\section{SPLITTING AND FIBRE-TYPE GROUPING}

Fibre-type grouping is regarded as a phenomenon found exclusively in neurogenic disorders (Dubowitz and Brooke, 1973). In the neurogenic disorders, increased fibre density found in single fibre recordings can be correlated with histochemical fibre type grouping (Schwartz et al., 1977). In addition, histological studies of the terminal motor innervation in these disorders, using the supravital methylene blue technique, have shown an increased terminal innervation ratio, and the presence of two or more motor endplates on single muscle fibres (Coërs et al., 1973). Fibre-type grouping is thus usually taken as strong evidence of reinnervation by axonal sprouting. However, small groups of fibres of similar histochemical type also occur in the myopathies (Ringel et al., 1976), including Duchenne muscular dystrophy (Schwartz et al., 1977) and polymyositis (Ringel et al., 1976). The late motor unit components (Desmedt and Borenstein, 1976) and increased fibre density found in these myopathies with single fibre EMG also indicate that enlargement of the motor unit has occurred (Schwartz et al., 1977).

There are several possible mechanisms which might account for enlargement of motor units in the myopathies. Firstly, small clusters of regenerating fibres must receive innervation by axonal sprouting in order to become functional. This phenomenon has been studied in regenerating fibres after experimental crush injuries (Reznik and Engel, 1970), and after ischaemic necrosis (Allbrook and Aitken, 1951).

Secondly, segmental fibre necrosis may separate a viable part of a fibre from its innervation (Denny-Brown, 1960). This denervated part of a fibre will then develop extrajunctional acetylcholine receptors (Katz and Miledi, 1964; Ringel et al., 1976). Either denervation atrophy (Hall-Craggs, 1971), or reinnervation by axonal sprouting from a nearby motor unit will follow (Miledi, 1962). Functional reinnervation of this denervated portion of a fibre can also occur if regenerating myotubes derived from the damaged segment re-establish continuity between the denervated and innervated parts of the fibre (Reznik, 1973), or with split or otherwise damaged, but innervated, nearby fibres. The continuity of the basement membrane scaffold of the damaged fibre (Vracko, 1974) will determine which of these processes occurs.

Thirdly, longitudinal fibre splitting can lead to forking of individual fibres. In serial sections 


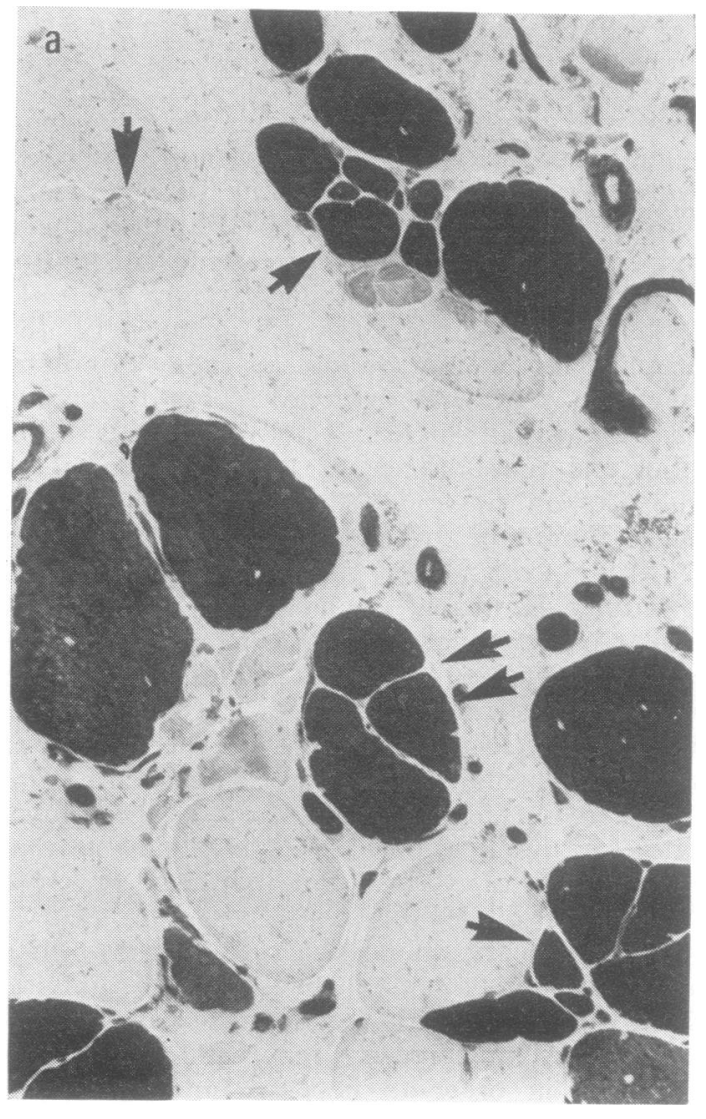

(Fig. 2), the separated parts, which retain functional innervation through their connection with their parent fibre, often become larger in cross-sectional area, resulting in a compensatory increase in myofibrillar mass, and in an apparent increase in the number of fibres in individual motor units. In random transverse sections this phenomenon may sometimes resemble fibre type grouping (Fig. 2), and in single fibre EMG recordings it is one factor leading to an increased fibre density, a phenomenon observed in both neurogenic (Stålberg et al., 1975; Schwartz et al., 1976) and myopathic disorders (Stålberg et al., 1974; Schwartz et al., 1977). The forked branches themselves may insert in the perimysium of adjacent fibres, fuse with their parent fibres, or continue for variable distances through the muscle belly before ending blindly (Isaacs et al., 1973; Schwartz et al., 1976). Ekstedt and Stålberg (1969) have suggested that forked fibres can be recognised with single fibre EMG by the finding of an

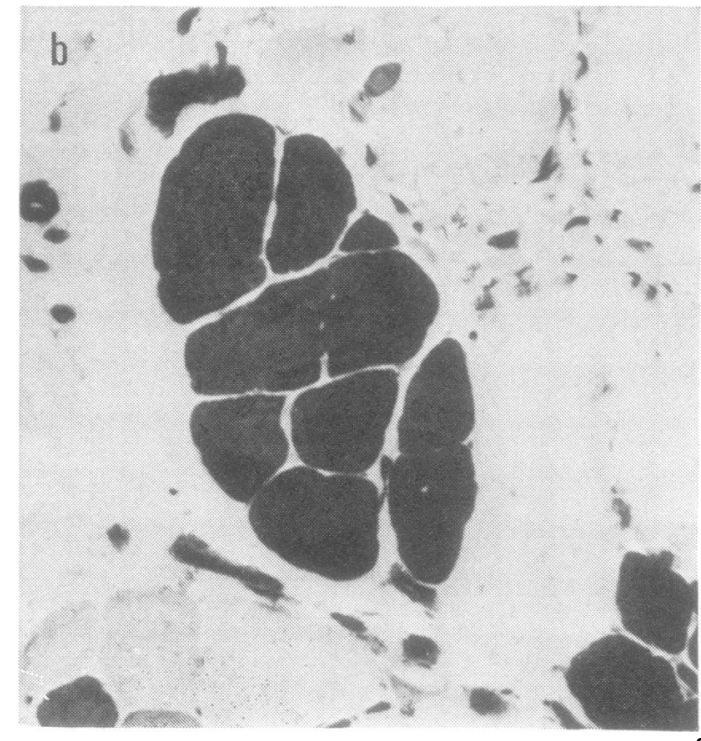

Fig. 4 Kugelberg-Welander disease: same biopsy as Fig. 2. ATPase. pH $4.3 \times 140$. (a) Several clusters of fibres of similar histochemical type, but of variable size, have been formed by splitting (arrows). One cluster (double arrows) is clearly subendomysial; (b) A similar, larger cluster. In this section the origin of this cluster of type 1 fibres is uncertain, but serial transverse sections showed that they were formed by splitting from a single fibre.

abnormally low neuromuscular jitter between two action potentials belonging to the same motor unit, but this seems to be a very rare phenomenon (Schwartz et al., 1976).

Fourthly, longitudinal fibre splitting may result in the formation of completely separate fibres. At first these fibres will lack innervation, but if axonal sprouting and new endplate formation occur the split fibres will become functional components of an enlarged motor unit. These newly formed fibres will be shorter than normal fibres and will vary in cross-sectional area. They will probably arise and insert from the perimysium of adjacent fibres. In transverse sections this phenomenon can usually be recognised by the presence of clusters of fibres of similar histochemical type, but of varying size (Fig. 2). Split fibres may change their histochemical type in response to their new innervation but this will only very rarely be recognisable (Aloisi et al., 1974; Schwartz et al., 1976). 


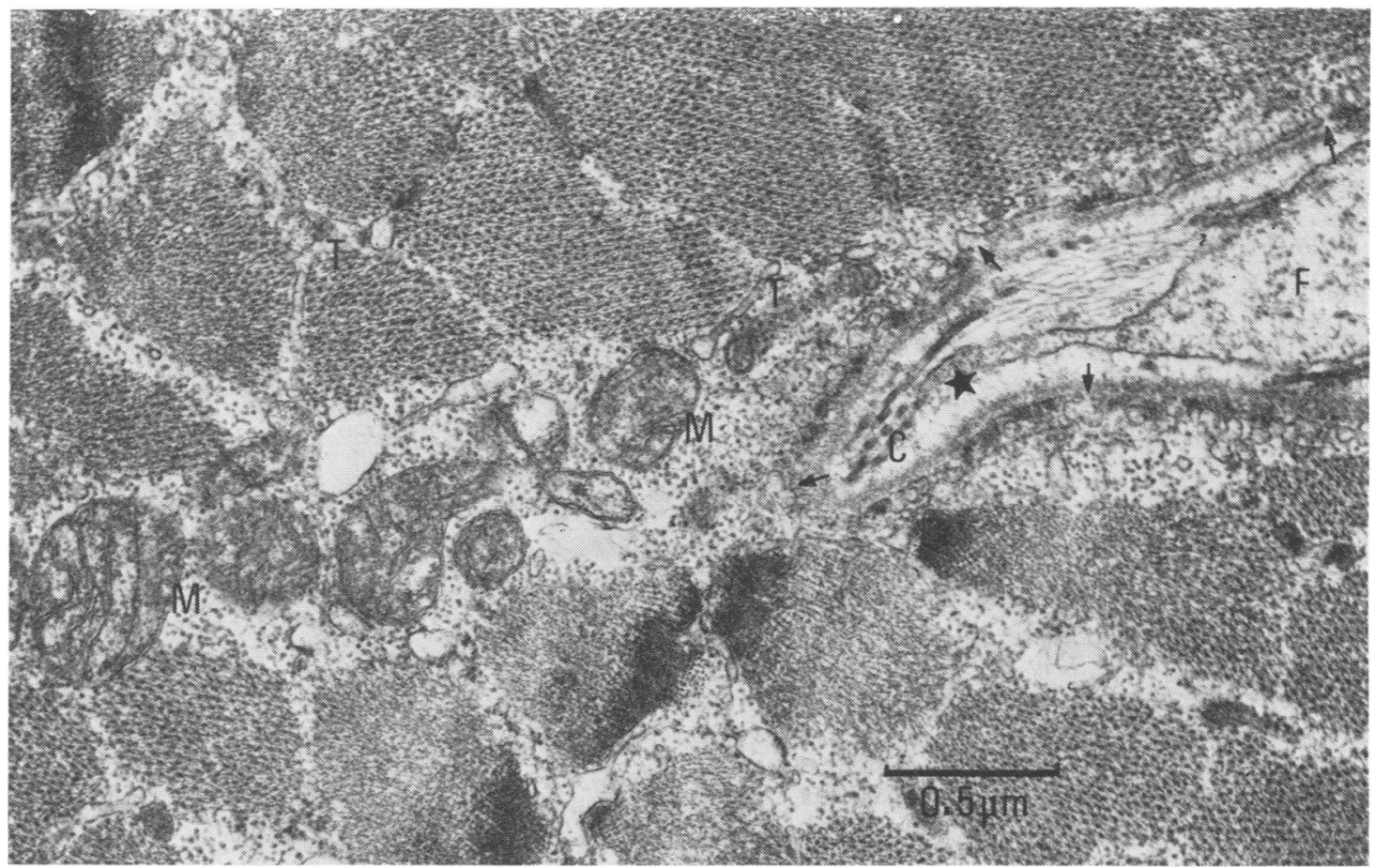

Fig. 5 Electron micrograph, transverse section. Fibre splitting in Kugelberg-Welander disease: same biopsy as Fig. 1. A fissure, marked by a line of mitochondria $(M)$, extends from the advancing edge of a cleft $\left(^{*}\right)$, separating the fibre into two parts. The plasma membrane lining the cleft is invaginated (arrows) by pinocytotic vesicles (cf Schwartz et al., 1976). The cleft contains a few collagen fibrils (c), and a fibroblast $(F) . T=$ tubules of the sarcoplasmic reticulum.

SPLITTING AS A COMPENSATORY PROCESS

In biopsies of longstanding neurogenic disorders secondary 'myopathic' abnormalities are common (Drachman et al., 1967; Schwartz et al., 1976). Fibre splitting is an important factor in the development of these abnormalities (Schwartz et al., 1976). It may result in an increase in the number of fibres and thus of myofibrillar mass in the weakened muscle, or it may lead to the formation of small non-viable fragments and thus to failure of compensation. In Duchenne muscular dystrophy EMG evidence of late components (Desmedt and Borenstein, 1975, 1976) with a markedly increased fibre density, and histochemical fibre type grouping, are all most prominent in the intermediate stages of the disease (Schwartz et al., 1977). In addition, Bell and Conen (1968) noticed that longitudinal fibre splitting was most common at this stage, when mobility is still possible. Later in the course of the disease compensation fails to keep pace with the progress of the disease, perhaps because regenera- tion fails, but also because continual fibre splitting results in the formation of fragments of insufficient size to become functional. Reinnervation of fibre fragments, or of regenerating fibres, may also be less successful at this stage. Reinnervation could be impaired because increasing interstitial fibrosis acts as a physical barrier to axonal sprouts, because there is less muscle fibre regeneration, or because split or regenerating fibres may be unable to attain a sufficient size to provide a suitable stimulus to attract innervation, or to form endplates. These factors are probably important in the rapid decompensation that occurs in the later stages of the disease.

In histological studies of the motor innervation in Duchenne muscular dystrophy, fine, beaded subterminal nerve fibres, forming small irregular endings on 'atrophic' fibres, have been found, but the terminal innervation ratio is normal (Coërs et al., 1973). Further histological work is needed to resolve the apparent conflict between this observation and the other histological 
(Schwartz et al., 1977) and physiological evidence (Borenstein and Desmedt, 1973; Desmedt and Borenstein, 1975, 1976; Schwartz et al., 1977).

In healed, longstanding polymyositis large polyphasic motor unit potentials of increased duration are often recorded (Mechler, 1974), and in such patients fibre type grouping is seen (Ringel et al., 1976). We have found similar EMG and histochemical findings in chronic polymyositis and, in addition, we have noted that fibre splitting is present (Swash et al., 1977). Fibre splitting may thus also be a contributory factor leading to fundamental compensation in polymyositis.

In biopsies of chronic or slowly progressive neurogenic disorders fibre hypertrophy with fibre splitting and scattered regenerating fibres (Drachman et al., 1967; Schwartz et al., 1976) are common features, and these lead to random variability in fibre size giving a myopathic appearance. These features probably represent a compensatory process leading to an increase in myofibrillar mass of functionally innervated fibres within enlarged motor units. However, in very longstanding neurogenic disorders, for example in long survivals after poliomyelitis, this secondary 'myopathic' change may itself lead to functional decompensation, as suggested by Drachman et al. (1967).

\section{Conclusion}

The role of longitudinal fibre splitting in progressive or longstanding neuromuscular disorders has been relatively neglected, although splitting was first recognised nearly a century ago (Erb, 1891). It deserves more attention, however, because it offers an explanation for many of the conflicting features of histological and electrophysiological investigations in the myopathies themselves (Borenstein and Desmedt, 1975; Workshop, 1975), and of the secondary 'myopathic' changes found in chronic neurogenic disorders (Schwartz et al., 1976). Further, it is one of several factors leading to functional compensation in the natural history of both neurogenic and myopathic disorders.

\section{References}

Adams, R. D., Denny-Brown, D., and Pearson, C. M. (1962). Diseases of Muscle. 2nd Edition. Henry Kimpton: London.

Allbrook, D. B., and Aitken, J. T. (1951). Reinnervation of striated muscle after acute ischaemia. Journal of Anatomy (London), 85, 376-390.

Aloisi, M., Pierobon Bormioli, S., and Schiaffino, S. (1974). Cell multiplication and histochemical changes in compensatory muscle hypertrophy. In
Structure and Function of Normal and Diseased $Z$ Muscle and Peripheral Nerve. Edited by I. Hausmanowa-Petrusewicz and H. Jedrejowska. Pp. 27-32. Polish Medical Publishers: Warsaw.

Banker, B. Q. (1960). The experimental myopathies. Research Publications. Association for Research in 0 Nervous and Mental Diseases, 38, 197-233.

Bell, C. D., and Conen, P. E. (1968). Histopathological $\stackrel{\bigcirc}{\supset}$ changes in Duchenne muscular dystrophy. Journal of the Neurological Sciences, 7, 529-544.

Borenstein, S., and Desmedt, J. E. (1973). Electro- $\overrightarrow{\overline{\bar{S}}}$ myographical signs of collateral reinnervation. In $\stackrel{\vec{\sim}}{\vec{D}}$ New Developments in Electromyography and Clini- 믈 cal Neurophysiology. Edited by J. E. Desmedt. Vol. 1, pp. 130-140. Karger: Basel.

Cazzato, G. (1970). Myopathic changes in denervated muscle: a study of biopsy material in various neuromuscular diseases. In Muscle Diseases. Edited by J. N. Walton, N. Canal, and G. Scarlato. Inter- $\vec{\circ}$ national Congress Series No. 199, pp. 392-401. Excerpta Medica: Amsterdam.

Coërs, C., Telerman-Toppet, N., and Gerard, J. M. (1973). Terminal innervation ratio in neuromuscular disease: Disorders of lower motor neurone, peripheral nerve and muscle. Archives of Neurolog 8 (Chicago), 29, 215-222.

Denny-Brown, D. (1960). Experimental studies per- $\stackrel{\oplus}{N}$ taining to hypertrophy, regeneration and degenera $\overrightarrow{\vec{C}}$ tion. Research Publications. Association fo⿱ $\mathrm{N}$ Research in Nervous and Mental Diseases, 38, $147 \mathrm{~T}$ 196.

Desmedt, J. E., and Borenstein, S. (1973). Collateraํㅕㅇ innervation of muscle fibres by motor axons of dystrophic motor units. Nature, 246, 500-501.

Desmedt, J. E., and Borenstein, S. (1975). Spontane ous fibrillation potentials in human muscula dystrophy: relation to muscle fibre segmentation? Nature, 258, 531-534.

Desmedt, J. E., and Borenstein, S. (1976). Regenera- . tion in Duchenne muscular dystrophy: electromyographic evidence. Archives of Neurology (Chicago), 33, 642-650.

Drachman, D. B., Murphy, J. R. N., Nigam, M. P., and Hills, J. R. (1967). 'Myopathic' changes in 음 chronically denervated muscle. Archives of Neurology (Chicago), 16, 14-24.

Dubowitz, V., and Brooke, M. H. (1973). Muscle Biopsy-a Modern Approach. Saunders: London.

Ekstedt, J., and Stålberg, E. (1969). Abnormal connections between skeletal muscle fibres. Electroencephalography and Clinical Neurophysiology, 27, 607-609.

Erb, W. H. (1891). Dystrophia muscularis progressiva. Deutsche Zeitschrift für Nervenheilkunde, 1, 13-94 and 173-261.

Haase, G. R., and Shy, G. M. (1960). Pathological changes in muscle biopsies from patients with peroneal muscular atrophy. Brain, 83, 631-637.

Hall-Craggs, E. C. B. (1971). Observations on the fate $\frac{D}{2}$ of muscle fibres temporarily isolated by transection of a muscle belly. Zeitschrift für Zellforschung und $\widetilde{N}$ Mikroskopische Anatomie, 119, 68-76. 
Hall-Craggs, E. C. B., and Lawrence, C. A. (1970). Longitudinal fibre division in skeletal muscle: a light and electronmicroscopic study. Zeitschrift für Zellforschung und Mikroskopische Anatomie, 109, 481-494.

Isaacs, G. R., Bradley, W. G., and Henderson, G. (1973). Longitudinal fibre splitting in muscular dystrophy: a serial cinematographic study. Journal of Neurology, Neurosurgery, and Psychiatry, 36, 813-819.

James, N. T. (1973). Compensatory hypertrophy in the extensor digitorum longus muscle of the rat. Journal of Anatomy (London), 116, 57-65.

Katz, B., and Miledi, R. (1964). The development of acetylcholine sensitivity in nerve-free segments of skeletal muscle. Journal of Physiology, 170, 389396.

Mechler, F. (1974). Changing electromyographic findings during the chronic course of polymyositis. Journal of the Neurological Sciences, 23, 237-242.

Miledi, R. (1962). Induced innervation of endplatefree muscle segments. Nature, 193, 281-282.

Reznik, M. (1973). Current concepts of skeletal muscle regeneration. In The Striated Muscle. Edited by C. M. Pearson. Pp. 185-225. Williams and Wilkins: Baltimore.

Reznik, M., and Engel, W. K. (1970). Ultrastructural and histochemical correlations of experimental muscle regeneration. Journal of the Neurological Sciences, 11, 167-185.

Ringel, S. P., Bender, A. N., and Engel, W. K. (1976). Extrajunctional acetylcholine receptors: alterations in human and experimental neuromuscular diseases. Archives of Neurology (Chicago), 33, 751-758.
Schmalbruch, H. (1976). Muscle fibre splitting and regeneration in diseased human muscle. Neuropathology and Applied Neurobiology, 2, 3-20.

Schwartz, M. S., Moosa, A., and Dubowitz, V. (1977). Correlation of single fibre EMG and muscle histochemistry. Journal of the Neurological Sciences, 31, 369-378.

Schwartz, M. S., Sargeant, M. K., and Swash, M. (1976). Longitudinal fibre splitting in neurogenic muscular disorders: its relation to the pathogenesis of myopathic change. Brain, 99, 617-636.

Stålberg, E., Schwartz, M. S., and Trontelj, J. V. (1975). Single fibre electromyography in various processes affecting the anterior horn cell. Journal of the Neurological Sciences, 24, 403-415.

Stålberg, E., Trontelj, J. V., and Janko, M. (1974). Single fibre EMG findings in muscular dystrophy. In Structure and Function of Normal and Diseased Muscle and Peripheral Nerve. Edited by I. Hausmanowa-Petrusewicz and $\mathrm{H}$. Jedrezejowska. Pp. 185-190. Polish Medical Publishers: Warsaw.

Swash, M., Schwartz, M. S., and Sargeant, M. K. (1977). Pathogenesis of longitudinal fibre splitting in neuromuscular disease. Neuropathology and Applied Neurobiology. In press.

Vracko, R. (1974). Basal lamina scaffold-anatomy and significance for maintenance of orderly tissue structure. American Journal of Pathology, 77, 314346.

Workshop on the Aetiology of Muscular Dystrophy (1975). In Recent Advances in Myology. Edited by W. G. Bradley, D. Gardner-Medwin, and J. N. Walton. Pp. 148-188. Excerpta Medica: Amsterdam. 